\title{
Response of chromospheric lines to different periodic non-thermal electron beams
}

\author{
Jianxia Cheng ${ }^{1,2}$ and Mingde Ding ${ }^{3}$ \\ ${ }^{1}$ Key Laboratory of Planetary Sciences, Shanghai Astronomical Observatory, Shanghai 200030, \\ China \\ email: chengjx@shao.ac.cn \\ ${ }^{2}$ Key Laboratory of Solar Activity, National Astronomical Observatories, Chinese Academy of \\ Sciences, Beijing 100012, China \\ ${ }^{3}$ School of Astronomy \& Space Science, Nanjing University, Nanjing 210093, China \\ email: dmd@nju.edu.cn
}

\begin{abstract}
Solar flares produce radiations in very broad wavelengths. Spectra can supply us abundant information about the local plasma, such as temperature, density, mass motion and so on. Strong chromospheric lines, like the most studied $\mathrm{H} \alpha$ and Ca II $8542 \AA$ lines are formed under conditions of departures from local thermodynamic equilibrium in the lower atmosphere subject to flare heating. Understanding how these lines are formed is very useful for us to correctly interpret the observations. In this paper, we try to figure out the response of chromospheric lines heated by different periodic non-thermal electron beams. Our results are based on radiative hydrodynamic simulations. We vary the periods of electron beam injection from $1.25 \mathrm{~s}$ to 20 s. We compare the response times to different heating parameters. Possible explanations are discussed.
\end{abstract}

Keywords. solar flare, radiative transfer, numerical simulation

\section{Introduction}

It has been known for many years that solar flares may exhibit fast fluctuations in emissions at various wavelengths. Early studies were focused on radio and hard X-ray observations. Fluctuations in optical lines have also been studied using ground instruments (e.g. Wang et al. 2000). Such fluctuations can be attributed to small-scale magnetic reconnections in the corona. High-energy electrons are recognized to be responsible for the hard X-ray and microwave emissions. Kašparová et al. (2009) studied the effect of short-duration electron-beam pulses on the temporal evolution of hydrogen Balmer lines using the Flarix code. Different from hard X-rays, optical lines take a little more time to respond to the impulsive beam heating. Trottet et al. (2000) compared the $\mathrm{H} \alpha$ time profile with the hard X-ray emission. Their result indicates that the chromospheric part of the flare is subject to heating by nonthermal electrons. Wang et al. (2000) found high frequency fluctuations in $\mathrm{H} \alpha$ wing at the footpoint which is spatially correlated with the hard X-ray source. Ding et al. (2001) performed numerical calculations to explain the fast variations of the $\mathrm{H} \alpha$ wing emission. In this paper, we study the response of chromospheric lines from an atmosphere heated by different periodic nonthermal electron beams using radiative hydrodynamic simulations.

\section{Numerical Method}

We perform radiative hydrodynamics modeling using the code RADYN with application to solar flares as described in detail in Abbett \& Hawley (1999). The simulations are 


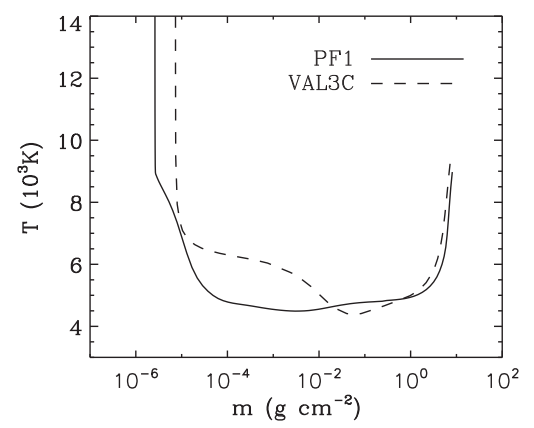

Figure 1. The base model, the PF1 model, adopted in the simulations. The VAL3C model is displayed for comparison.
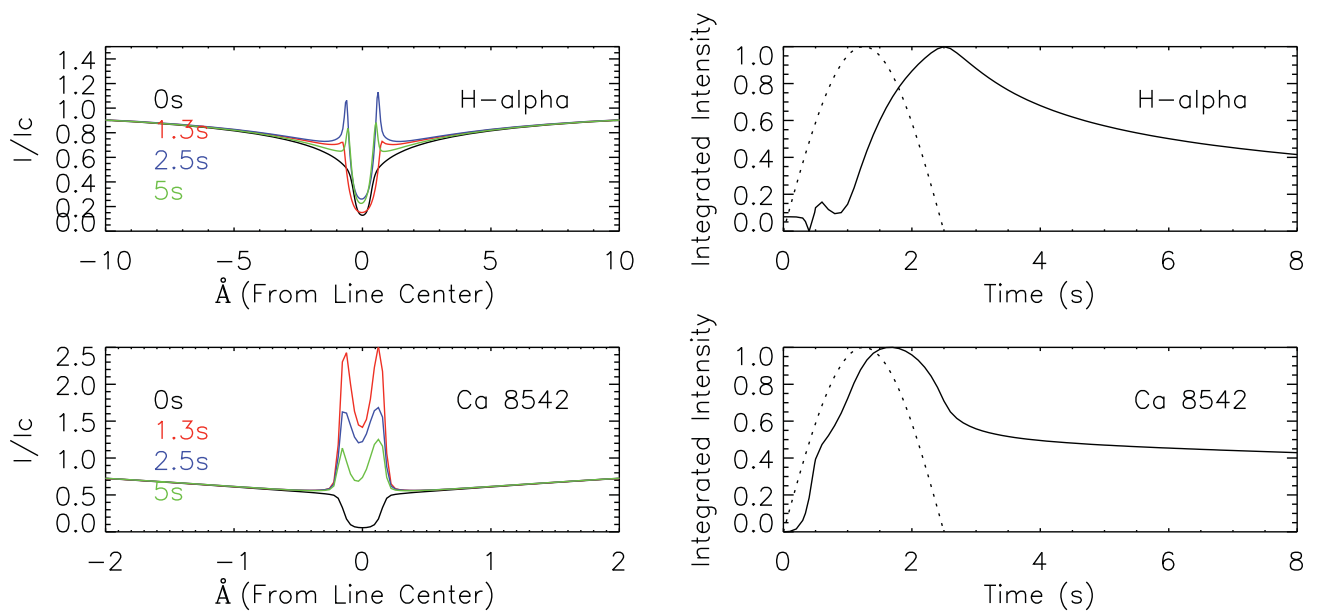

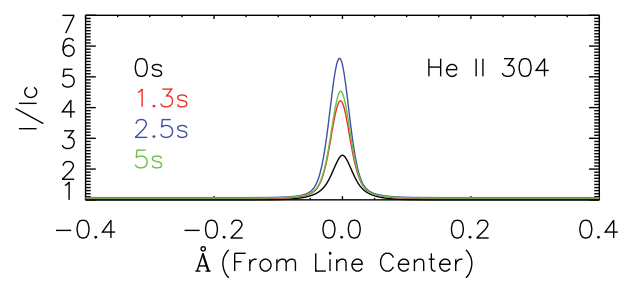

(a)

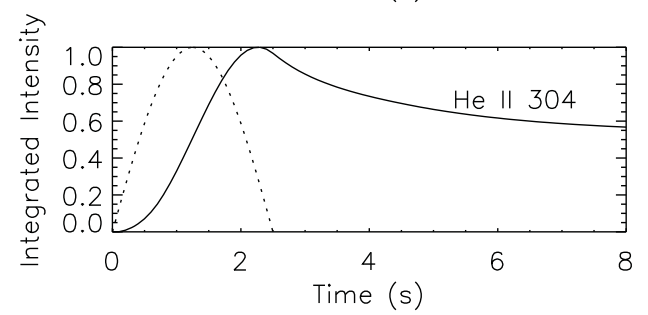

(b)

Figure 2. (a) Line profiles of three lines, $\mathrm{H} \alpha$, Ca 8542 and He $304 \AA$ from an atmosphere heated by an electron beam. Here, $\alpha=5 \mathrm{~s}$. (b) Normalized integrated intensities varying with time. Here, $\alpha=5 \mathrm{~s}$. The dashed lines refer to the electron beam flux.

started with the PF1 initial atmospheric model (Figure 1). The atmosphere is heated by a non-thermal electron beam which is assumed to have a power-law distribution with a spectral index $\delta=4$ and low energy cutoff $E_{c}=40 \mathrm{keV}$. The electron flux is assumed to vary sinusoidally with time as $F(t)=F_{0} \sin (2 \pi t / \alpha)$, where $F_{0}=10^{10} \mathrm{ergs} \mathrm{cm}^{-2} \mathrm{~s}^{-1}$, and $\alpha=2.5 \mathrm{~s}, 5 \mathrm{~s}, 10 \mathrm{~s}, 20 \mathrm{~s}$, and $40 \mathrm{~s}$. The electron beam heating lasts for a time period of $\alpha / 2$. 

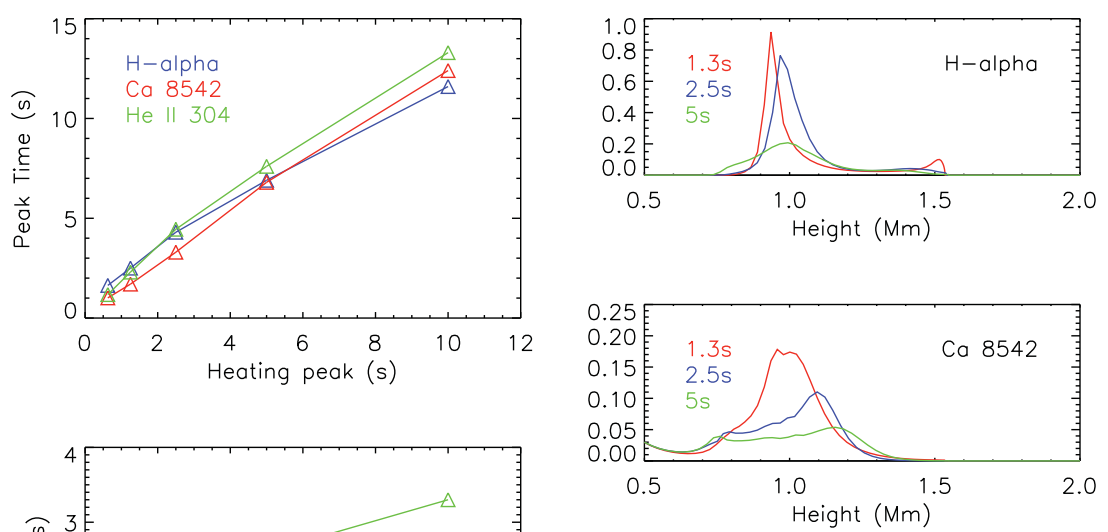

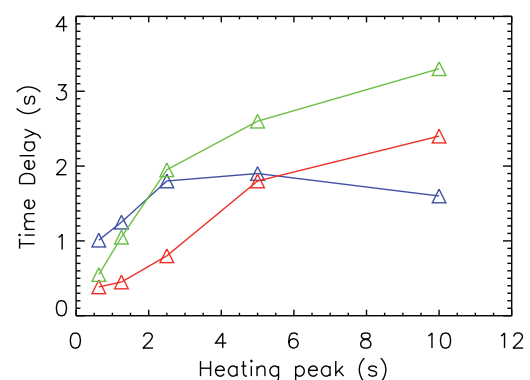

(a)

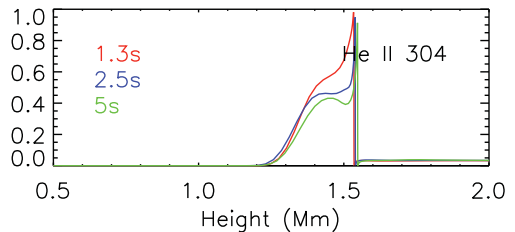

(b)

Figure 3. (a) Peaking times and time delays of integrated intensities varying with different heating peaks $(\alpha / 4)$. (b) Contribution functions at line wings varying with height. Here, $\alpha=5$ s.

\section{Computations and Results}

Here, we take the case of $\alpha=5 \mathrm{~s}$ as an example to show the spectral line evolutions in three different lines (Figure 2a). As the non-thermal heating continues, the intensities at all the three wavelengths show some enhancements. For the $\mathrm{H} \alpha$ line, the intensity shows a slight decrease at the line center firstly, and then starts to increase. A central reversal occurs at the same time. The He II $304 \AA$ line shows a very significant enhancement during the non-thermal heating. In general, all the three lines show some enhancements when the atmosphere is heated by non-thermal electrons although their peak times and variations show some differences. Furthermore, we compare the integrated intensities with the non-thermal electron fluxes directly (Figure 2b). Time delays between the peak of integrated intensity and the heating rate peak are very obvious. Based on the calculations above, we vary the heating duration as $\alpha / 2=1.25 \mathrm{~s}, 2.5 \mathrm{~s}, 5 \mathrm{~s}, 10 \mathrm{~s}, 20 \mathrm{~s}$ to check how these lines respond to this parameter. The results are displayed in Figure 3a. It is seen that the peak times of integrated intensity are correlated well with those of the nonthermal heating rate. The bottom panel of Figure 3a shows the time delays of the line intensity peak relative to the heating rate peak. As the heating duration increases, the time delays become larger in most cases; the only exception is the $\mathrm{H} \alpha$ line, for which the time delays decrease when the heating duration lasts for 10 seconds $(\alpha=20 \mathrm{~s})$. The different responses of the three lines may be partly attributed to their different formation regions. Note that the peak time of the He II $304 \AA$ line is somewhat later than that of the Ca II $8542 \AA$ line in this case. Probably this is due to a relatively high low energy cutoff of the electron beam adopted here, which tends to deposit energy at a relatively lower height. The intensity contribution functions at line wings varying with heights are given in Figure 3b. Here, the heating parameter $\alpha=5$ s. As the electron 
beam heating continues, distributions of the contribution functions vary slightly. In fact, the line formation regions are very complicated because of the complexity in the atomic transition processes which are seriously affected by the local atmospheric conditions. Line center and wings should be formed in different atmospheric layers.

\section{Acknowledgements}

This work is supported by NSFC under grants 11303073 ,11373023,11133004, 11103008, and the strategic priority research program of the Chinese Academy of Sciences (Grant No. XDB09000000). This research is also supported by the open project of Key Laboratory of Solar Activity, National Astronomical Observatories, Chinese Academy of Sciences.

\section{References}

Abbett, W. P. \& Hawley, S. L. 1999, ApJ, 521, 906

Ding, M. D., Qiu, J., Wang, H., \& Goode, P. R. 2001, ApJ, 552,340

Kašparová, J., Varady, M., Heinzel, P., Karlicky, M., \& Moravec, Z. 2009, Aש̋A, 499, 923

Trottet, G., et al. 2000, A\&SA, 356, 1067

Wang, H., Qiu, J., Denker, C., Spirock, T., Chen, H., \& Goode, P. R. 2000, ApJ, 542, 1080 